\title{
Cosmological evolution of orientations of cluster-sized dark matter haloes and their central galaxies in the Horizon-AGN simulation
}

\author{
Taizo Okabe ${ }^{1}{ }^{\star}$ Takahiro Nishimichi ${ }^{2,3}$, Masamune Oguri ${ }^{1,2,4}$, Sébastien Peirani ${ }^{5,6}$, \\ Tetsu Kitayama ${ }^{7}$, Shin Sasaki ${ }^{8}$, Yasushi Suto ${ }^{1,4}$, Christophe Pichon ${ }^{6,9}$, \\ and Yohan Dubois 6 \\ ${ }^{1}$ Department of Physics, The University of Tokyo, 7-3-1 Hongo, Bunkyo-ku, Tokyo 113-0033, Japan \\ ${ }^{2}$ Kavli Institute for the Physics and Mathematics of the Universe (WPI), The University of Tokyo Institutes for Advanced Study, \\ The University of Tokyo, 5-1-5 Kashiwanoha, Kashiwa, Chiba 277-8583, Japan \\ ${ }^{3}$ Center for Gravitational Physics, Yukawa Institute for Theoretical Physics, Kyoto University, Kyoto 606-8502, Japan \\ ${ }^{4}$ Research Center for the Early Universe, School of Science, The University of Tokyo, 7-3-1 Hongo, Bunkyo-ku, Tokyo, 113-0033, Japan \\ ${ }^{5}$ Université Côte d'Azur, Observatoire de la Côte d'Azur, \\ CNRS, Laboratoire Lagrange, Bd de lâĂŹObservatoire, CS 34229, 06304 Nice Cedex 4, France \\ ${ }^{6}$ Institut d'Astrophysique de Paris (UMR 7095: CNRS \& UPMC), 98 bis Bd Arago, 75014 Paris, France \\ ${ }^{7}$ Department of Physics, Toho University, Funabashi, 2-2-1 Miyama, Funabashi, Chiba 274-8510, Japan \\ ${ }^{8}$ Department of Physics, Tokyo Metropolitan University, 1-1 Minami-Osawa, Hachioji, Tokyo 192-0397, Japan \\ ${ }^{9}$ Korea Institute for Advanced Study, 85 Hoegiro, Dongdaemun-gu, Seoul, 02455, Republic of Korea
}

Accepted XXX. Received YYY; in original form ZZZ

\begin{abstract}
It is known observationally that the major axes of galaxy clusters and their brightest cluster galaxies are roughly aligned with each other. To understand the origin of the alignment, we identify 40 cluster-sized dark matter $(\mathrm{DM})$ haloes with masses higher than $5 \times 10^{13} M_{\odot}$ and their central galaxies (CGs) at $z \approx 0$ in the Horizon-AGN cosmological hydrodynamical simulation. We trace the progenitors at 50 different epochs between $0<z<5$. We then fit their shapes and orientations with a triaxial ellipsoid model. While the orientations of both DM haloes and CGs change significantly due to repeated mergers and mass accretions, their relative orientations are well aligned at each epoch even at high redshifts, $z>1$. The alignment becomes tighter with cosmic time; the major axes of the CGs and their host DM haloes at present are aligned on average within $\sim 30^{\circ}$ in the three dimensional space and $\sim 20^{\circ}$ in the projected plane. The orientations of the major axes of DM haloes on average follow one of the eigen-vectors of the surrounding tidal field that corresponds to the slowest collapsing (or even stretching) mode, and the alignment with the tidal field also becomes tighter. This implies that the orientations of CGs and DM haloes at the present epoch are largely imprinted in the primordial density field of the Universe, whereas strong dynamical interactions such as mergers are important to explain their mutual alignment at each epoch.
\end{abstract}

Key words: methods: numerical - galaxies: clusters: general - dark matter

\section{INTRODUCTION}

Observed shapes of galaxies and galaxy clusters are not spherical, but rather approximated well by ellipsoids. Their orientations defined by the position angles of the major axes may indicate a preferred direction in their formation process

^ E-mail: taizo.okabe@utap.phys.s.u-tokyo.ac.jp that is supposed to reflect the initial condition and/or the dynamical evolution.

There are numerous observational studies that have reported statistical correlations of those orientations over various scales. One of the most well-known results is the alignment between the orientations of the brightest cluster galaxies (BCGs) and their host clusters (e.g. Sastry 1968; Carter \& Metcalfe 1980; Binggeli 1982). For instance, Binggeli 
(1982) reported that merger axes of 39 galaxy clusters at $z<0.1$ and their BCGs are aligned with $\sim 30^{\circ}$ on average. This result has been studied further and confirmed for wider samples at different redshifts (e.g. Wang et al. 2008; Panko et al. 2009; Hao et al. 2011; Huang et al. 2016).

More recently, West et al. (2017) measured the alignment between orientations of clusters and their BCGs, and obtained a mean value of about $\sim 30^{\circ}$ for 52 clusters. Their most important finding is that the alignment extends to $z>1.3$ with high statistical significance. While the alignments are ubiquitous observationally, their physical origin is not well understood, and remains to be explained theoretically.

Cosmological (dark matter only) $N$-body simulations have been used for understanding the origin of those observed alignments. For instance, there have been many previous attempts to examine the alignment among the major axes of dark matter (DM) haloes at different scales (e.g. West et al. 1991; West 1994; Dubinski 1998; Jing \& Suto 2002; Faltenbacher et al. 2008). In particular, Jing \& Suto (2002) introduced triaxial modeling of dark matter haloes in the cold dark matter (CDM) model, and found that major axes of iso-density surfaces at different density thresholds in the same halo are roughly aligned. Suto et al. (2016) further examined the evolution of DM haloes, and found that shapes and position angles of the inner regions change significantly over the cosmic time relative to the outer region of the same cluster-sized haloes. They also found that around $z=0$, the inner region of those haloes become rounder than the outer region, and tend to be aligned toward the orientation of the host DM halo (see their Figure 4).

Since $N$-body simulations do not include baryon physics, BCGs cannot be defined in a straightforward manner. It is not clear to what extent the orientation of the inner region of those DM haloes can be regarded as a good proxy for that of BCGs. Reliable predictions concerning the alignment between the orientations of BCGs and their hosting DM haloes require cosmological hydrodynamical simulations that incorporate proper baryon physics including gas cooling, star formation, and supernova/AGN feedback as well. Several previous attempts (e.g. Dong et al. 2014; Velliscig et al. 2015; Tenneti et al. 2015; Chisari et al. 2017) have found that major axes of BCGs and their host DM haloes are fairly well aligned, although the result should depend on how to implement baryon physics in a reliable fashion.

We are carrying out systematic studies of the nonsphericity and orientation of galaxy clusters using the Horizon-AGN simulation (Dubois et al. 2014), a state-ofthe-art cosmological hydrodynamical simulation incorporating proper baryon physics. Suto et al. (2017) (Paper I) focused on the projected-axis ratios of the stellar component, X-ray-emitting gas, and DM in 40 cluster-sized simulated DM haloes with $M_{\mathrm{DM}}>5 \times 10^{13} M_{\odot}$, and showed that even shapes of DM haloes in the outer region of clusters are substantially affected by baryon physics. Indeed, the projected axis ratios of the simulated haloes become consistent with those derived from the observed X-ray clusters only when the AGN feedback is included.

Okabe et al. (2018) (Paper II) computed the position angles of DM, gas, and stellar mass distributions for those 40 haloes at the present epoch, $z \approx 0$ alone, and examined the statistics of their mutual alignment. In particular, $\mathrm{Pa}$ - per II examined the difference of the position angles $\Delta \theta$ of various components relative to that of the $\mathrm{CG}$ in the same halo, and found that the root mean square of $\Delta \theta$ is less than $25^{\circ}$, indicating that they are relatively well aligned with each other. While this conclusion is consistent with both previous simulations and observational results, the origin of the alignment is not yet clear. In this paper, we extend Paper II and attempt to explain the origin by considering the evolution of the alignment.

Furthermore, there are many recent studies that report the alignment of clusters over $\sim 100 h^{-1} \mathrm{Mpc}$ scales, and also between galaxies/clusters and the large-scale structure surrounding them (e.g. Catelan et al. 2001; Hirata \& Seljak 2004; Patiri et al. 2006; Hirata et al. 2007; Brunino et al. 2007; Blazek et al. 2011; Smargon et al. 2012; Codis et al. 2015; Blazek et al. 2015; Chen et al. 2016; van Uitert \& Joachimi 2017; Okumura et al. 2017; Chisari et al. 2017; van Uitert \& Joachimi 2017; Blazek et al. 2017; Osato et al. 2018; Piras et al. 2018; Codis et al. 2018; Chen et al. 2019; Durret et al. 2019). This raises the possibility that the orientation of DM haloes is imprinted in the large-scale structure in the Universe and BCGs tend to be aligned dynamically toward a particular direction. Of course this picture may be over-simplified and should be tested quantitatively against numerical simulations. This is exactly what we attempt in the present paper.

Note that a companion paper, Bate et al. (2019), performs a related analysis on the Horizon-AGN simulation, extending a comprehensive study of intrinsic alignments by Chisari et al. (2017). While their main interest lies in the origin of the alignment between elliptical galaxies and the large-scale structure of the Universe, we investigate in the present paper the alignments between BCGs and the tidal fields to understand the origin of the orientations and alignments between BCGs and their host DM haloes. Therefore, the purposes of our work and their work are different, although methodology of the analysis is of course similar.

The structure of this paper is as follows. Section 2 first presents a brief summary of our identification scheme of central galaxies (CGs), simulated counterparts of the observed BCGs, and their hosting DM haloes from the Horizon-AGN simulation. We also describe how to estimate the orientation of those objects from an ellipsoidal fit using a mass tensor, and the tidal field of the large-scale structure. A representative example for one particular simulated cluster is shown in Section 3, and the statistical analysis over 40 haloes are presented in Section 4. Finally, Section 5 is devoted to the summary of the paper.

The Horizon-AGN simulation adopts the following cosmological parameters. The total matter density $\Omega_{m}=0.272$, the baryon density $\Omega_{b}=0.045$, the dark energy density $\Omega_{\Lambda}=0.728$, the dimensionless Hubble parameter $h=0.704$, the amplitude of the power spectrum of density fluctuations averaged over the sphere of $8 h^{-1} \mathrm{Mpc}$ radius at present epoch $\sigma_{8}=0.81$, the power-law index of the primordial power spectrum $n_{s}=0.967$. We also adopt the same cosmological parameters throughout the paper. 


\section{ORIENTATIONS OF DARK MATTER HALOES, CENTRAL GALAXIES, AND TIDAL FIELDS IN THE HORIZON-AGN SIMULATION}

The Horizon-AGN simulation (Dubois et al. 2014, Papers I and II) follows the evolution of three components, DM, star, and gas. DM and stars are represented by collisionless particles, whereas gas components are assigned on meshes in the simulation box and solved with the adaptive mesh refinement. While Papers I and II examined the orientation and ellipticity of all the three components, this paper focuses on the relation between DM haloes and CGs, with application to weak lensing and galaxy surveys in mind. Thus, we do not consider the gas component in this paper. The HorizonAGN simulation has a box size of $\left(100 h^{-1} \mathrm{cMpc}\right)^{3}$, where cMpc denotes comoving Mpc. Since we consider both small $(\sim \mathrm{kpc})$ and large $(\sim \mathrm{Mpc})$ scales in this paper, we use both comoving and physical coordinates. The final mesh size at the densest region is about $\sim 1 h^{-1}$ ckpc. On the other hand, dynamics of collisionless dark matter and stellar particles is followed by the particles-mesh solver (Dubois et al. 2014).

Since our current study is entirely based on the HorizonAGN simulation, its reliability of baryon physics and the extent to which it reproduces the empirical nature of galaxies and clusters are crucially important. It has found to be in good agreement, with a number of observed properties including the intrinsic alignment of galaxies (Chisari et al. 2015, 2016), density profile of massive galaxies (Peirani et al. 2017, 2019), cosmic star formation history over the redshift range $1<z<6$ (Kaviraj et al. 2017), morphological diversity of galaxies (Dubois et al. 2016), alignments between galactic spin and the nearest filament Welker et al. (2019) and the ellipticity distribution of X-ray galaxy clusters $(\mathrm{Pa}-$ per I). Therefore the Horizon-AGN simulation is supposed to be one of the best simulated datasets currently available for our purpose, even if not perfect.

\subsection{Identification of cluster-sized dark matter haloes and central galaxies}

Following Paper II, we use the ADAPTAHOP (Aubert et al. 2004; Tweed et al. 2009) to identify DM haloes and galaxies from DM and stellar particles, respectively, and select cluster-sized haloes with DM mass of $M_{\mathrm{DM}}>5 \times 10^{13} M_{\odot}$ at $z \approx 0$. ADAPTAHOP is a subhalo finder that separates multiple subhaloes while comparing the relative heights of peaks and saddle points of the smoothed density field. We select stellar haloes by applying the ADAPTAHOP to stellar particles. We define stellar haloes with more than 50 stellar particles as galaxies. Since each stellar particles has the mass of about $2 \times 10^{6} M_{\odot}$, this criterion corresponds to the minimum stellar mass of about $10^{8} M_{\odot}$ in our final galaxy catalog. In total, we have $N_{\mathrm{cl}}=40$ haloes that are identical to those analysed in Paper II, but we identify their progenitor haloes at 50 different redshifts so as to trace their evolution. The 50 epochs are selected from $z \sim 5(t \sim 1.5 \mathrm{Gyr})$ to $z \sim 0$ $(t \sim 13.5 \mathrm{Gyr})$ in an equal time interval of $\Delta t \sim 250 \mathrm{Myr}$. We make the merger trees of all the 40 cluster-sized DM haloes by using TREEMAKER (Tweed et al. 2009), which first builds the merger history tree, and then connects haloes with their progenitors.
Once DM haloes are identified at redshift 0 , we define the $\mathrm{CG}$ in each halo as the most massive galaxy in a halo within $1 \mathrm{pMpc}$ from the most bound particle of each halo (see Paper II, for more detail). Thus we define the CG at each epoch $t$ by using the CG in the previous epoch $t-\Delta t$. Specifically, we define the CG at each epoch $t$ as a galaxy containing the largest number of stellar particles of the CG in the adjacent snapshot $t-\Delta t$ and is located within $100 \mathrm{pkpc}$ from the most bound particle of each halo at each epoch $t$. We expect that the CG selected by the above procedure are similar to observed BCGs. Finally we define the "halo centre" by the centre-of-mass of the CG, instead of the centre-ofmass of the DM halo; see equation (2) below.

\subsection{Procedure of ellipsoid fit}

Once DM haloes and CGs are identified at each epoch, we fit them to the triaxial ellipsoid model in three-dimensional space (Paper I), and measure the major, intermediate, and minor axis vectors, $\hat{a}_{1}, \hat{a}_{2}$, and $\hat{a}_{3}$, respectively, unlike in Paper II that fit the data in the projected two dimensional space.

More specifically, we follow the ellipsoid fitting based on the inertia tensor as described in Suto et al. (2016). From all the star particles belonging to the CG, we first compute its centre-of-mass position $x_{\mathrm{CG}, \alpha}^{\mathrm{CM}}(\alpha=1,2,3)$, and compute the following mass tensor from the star particles located within a sphere of radius $20 \mathrm{pkpc}$ from $x_{\mathrm{CG}, \alpha}^{\mathrm{CM}}$ :

$I_{\mathrm{CG}, \alpha \beta}(z) \equiv \frac{\sum_{n=1}^{N_{\mathrm{star}}} m_{\mathrm{star}}^{(n)}\left[x_{\mathrm{star}, \alpha}^{(n)}-x_{\mathrm{CG}, \alpha}^{\mathrm{CM}}\right]\left[x_{\mathrm{star}, \beta}^{(n)}-x_{\mathrm{CG}, \beta}^{\mathrm{CM}}\right]}{\sum_{n=1}^{N_{\mathrm{star}}} m_{\mathrm{star}}^{(n)}}$,

where $m_{\mathrm{star}}^{(n)}$ and $x_{\mathrm{star}, \alpha}^{(n)}$ are the mass and the coordinate of the $n$-th stellar particle $\left(n=1, \cdots, N_{\text {star }}\right)$.

The above mass tensor is diagonalized and the directions of the major, intermediate, and minor axes are computed. We then select the size of the ellipsoid $R_{a b c}^{\text {star }} \equiv$ $\sqrt[3]{a_{1} a_{2} a_{3}}=20$ pkpc, where $a_{1}, a_{2}$, and $a_{3}$ are the half lengths of the major, intermediate, and minor axes, respectively. We repeat the above procedure using the star particles in the ellipsoid around the update centre-of-mass position $x_{\mathrm{CG}, \alpha}^{\mathrm{CM}}$. We choose the value of $20 \mathrm{pkpc}$ as the size of CGs for definiteness. We confirmed that changing the value to 10 or $30 \mathrm{pkpc}$ does not affect the main conclusion of this paper (see also Paper II).

The whole procedure is iterated until the three eigenvalues of the mass tensor converge within a fractional error of $10^{-8}$. We then redefine the $\mathrm{CG}$ as the set of star particles within the ellipsoid of $R_{a b c}^{\mathrm{star}}=20 \mathrm{pkpc}$, and characterize the CG by the parameters including the half lengths of major axis $a_{1}$, intermediate axis $a_{2}$, and minor axis $a_{3}$ $\left(a_{1} \geq a_{2} \geq a_{3}\right)$, their direction, and the centre of mass $x_{\mathrm{CG} \alpha}^{\mathrm{CM}}$. Therefore the resulting CG is different from the original set of star particles identified with the ADAPTAHOP halo finder.

The shape and orientation of the host DM halo at each $z$ are computed similarly except that we use the mass tensor of DM particles around the centre-of-mass of the CG:

$I_{\mathrm{DM}, \alpha \beta}(z) \equiv \frac{\sum_{n=1}^{N_{\mathrm{DM}}} m_{\mathrm{DM}}\left[x_{\mathrm{DM}, \alpha}^{(n)}-x_{\mathrm{CG}, \alpha}^{\mathrm{CM}}\right]\left[x_{\mathrm{DM}, \beta}^{(n)}-x_{\mathrm{CG}, \beta}^{\mathrm{CM}}\right]}{\sum_{n=1}^{N_{\mathrm{DM}}} m_{\mathrm{DM}}}$, 
where $m_{\mathrm{DM}}$ and $x_{\mathrm{DM}, \alpha}^{(n)}$ are the mass and the coordinate of the $n$-th dark matter particle within the ellipsoid. In this calculation, we use all the dark matter particles including those in subhaloes. Once we fix the size of the ellipsoid, $R_{a b c}^{\mathrm{DM}} \equiv \sqrt[3]{a_{1} a_{2} a_{3}}$, we can compute the total mass and number of DM particles within the ellipsoid, $M_{\mathrm{DM}}$ and $N_{\mathrm{DM}}$. Unlike in the case of $\mathrm{CG}$, we consider three values of the ellipsoidal bound so that the corresponding to $M_{\mathrm{DM}}=0.1 M_{200}, 0.5 M_{200}$, and $M_{200}$, where $M_{200}$ is the mass of a sphere whose average DM density is 200 times larger than the cosmic critical density at each $z$.

\subsection{The tidal field of the large-scale mass distribution}

As mentioned in Section 1, the orientations of DM haloes are correlated to their surrounding matter distribution. Let us expand the gravitational potential of the matter with respect to the centre of a DM halo, $x^{\mathrm{CM}}$ :

$$
\begin{aligned}
\Phi(\boldsymbol{x})= & \Phi\left(\boldsymbol{x}^{\mathrm{CM}}\right)+\sum_{\alpha=1}^{3}\left(x_{\alpha}-x_{\alpha}^{\mathrm{CM}}\right)\left(\frac{\partial \Phi}{\partial x_{\alpha}}\right)_{\boldsymbol{x}=\boldsymbol{x}^{\mathrm{CM}}} \\
& +\frac{1}{2} \sum_{\alpha, \beta=1}^{3}\left(x_{\alpha}-x_{\alpha}^{\mathrm{CM}}\right)\left(x_{\beta}-x_{\beta}^{\mathrm{CM}}\right)\left(\frac{\partial^{2} \Phi}{\partial x_{\alpha} \partial x_{\beta}}\right)_{\boldsymbol{x}=\boldsymbol{x}^{\mathrm{CM}}} \\
& +\cdots
\end{aligned}
$$

The third term in equation (3) describes the tidal field around the DM halo and is responsible for its ellipsoidal growth. If we define the tidal field tensor:

$T_{\alpha \beta}=\frac{\partial^{2} \Phi}{\partial x_{\alpha} \partial x_{\beta}}$

its eigen-vectors and eigen-values characterize the direction and relative growth rate of the ellipsoidal evolution of the object.

We compute the tidal field tensor from the simulation data as follows. We first divide the simulation box into $100^{3}$ small grids and assign the DM density field $\rho(\boldsymbol{x})$ at each grid by a cloud-in-cell interpolation with $\boldsymbol{x}$ being the comoving coordinates of the grid. Next we define the dimensionless density contrast fields:

$\delta(\boldsymbol{x})=\frac{\rho(\boldsymbol{x})-\langle\rho\rangle}{\langle\rho\rangle}$,

where $\langle\rho\rangle$ is the mean density averaged over the entire simulation box. Then the tidal tensor $T_{\alpha \beta}(\boldsymbol{x})$ at each grid is defined by the second spatial derivative of the smoothed density contrast. If we adopt a Gaussian smoothing over a scale $\sigma$, the Fourier transform of $T_{\alpha \beta}(\boldsymbol{x})$ is easily computed as

$\tilde{T}_{\alpha \beta}(\boldsymbol{k})=\frac{k_{\alpha} k_{\beta}}{|\boldsymbol{k}|^{2}} \tilde{\delta}(\boldsymbol{k}) \exp \left(\frac{-|\boldsymbol{k}|^{2} \sigma^{2}}{2}\right)$,

where $k_{\alpha}$ and $\tilde{\delta}(\boldsymbol{k})$ are $\alpha$-th component of the wave-number vector $\boldsymbol{k}$ and the Fourier transform $\delta(\boldsymbol{x})$, respectively. We use the FFTW package to compute the Fourier transform of the tidal field (Frigo \& Johnson 1998; Frigo 1999; Frigo \& Johnson 2005).

Since the spatial extent of cluster-sized haloes is typically $\sim 1 h^{-1} \mathrm{cMpc}$, we choose $\sigma=3,5$, and $10 h^{-1} \mathrm{cMpc}$ as the smoothing scale so that the corresponding tidal tensor traces the large-scale structure surrounding those haloes. Then, we compute the inverse Fourier transform of $\tilde{T}_{\alpha \beta}(\boldsymbol{k})$ to obtain the tidal tensor $T_{\alpha \beta}(\boldsymbol{x})$. We apply the cloud-in-cell interpolation of the tidal tensors at the nearby grids to obtain the tidal tensor defined at the centre of the CG that is assumed to be the centre of the host DM halo as well.

Finally, we diagonalize the tidal field tensor to obtain the normalized eigenvectors, $\hat{u}_{\alpha}(\alpha=1,2$, and 3$)$, and the corresponding eigenvalues with $\lambda_{1}, \lambda_{2}$, and $\lambda_{3}$ of $\lambda_{1} \geq \lambda_{2} \geq \lambda_{3}$. In particular, $\hat{u}_{3}$ corresponds to the direction of the slowest collapsing or the fastest expanding mode, and expected to be correlated to the major axis of the object located at the centre. Previous studies (e.g., Hahn et al. 2007; Lee 2019) found that the set of eigenvalues roughly corresponds to the structure defined at the location as follows;

(i) clusters $\left(\lambda_{1}>0, \lambda_{2}>0\right.$, and $\left.\lambda_{3}>0\right)$,

(ii) filaments $\left(\lambda_{1}>0, \lambda_{2}>0\right.$, and $\left.\lambda_{3}<0\right)$,

(iii) sheets $\left(\lambda_{1}>0, \lambda_{2}<0\right.$, and $\left.\lambda_{3}<0\right)$,

(iv) voids $\left(\lambda_{1}<0, \lambda_{2}<0\right.$, and $\left.\lambda_{3}<0\right)$.

We confirmed that for $\sigma=10 h^{-1} \mathrm{cMpc} 11$ of our haloes are classified as "clusters", and the remaining 29 haloes are as "filaments" according to the above classification.

\section{AN EXAMPLE OF ELLIPSOID FIT}

In this section, we select the most massive single-coredominated halo from the 40 haloes in our sample, which is the same as plotted in Figure 3 of Paper I. Figure 1 shows the evolution of the mass of the DM halo and CG (top), the ratios of their major and minor axes $a_{3} / a_{1}$ (middle), and the angles between their major axes (bottom). The masses of the DM halo and CG are plotted in blue and red, respectively, in the top panel. The axis ratios, $a_{3} / a_{1}$, are computed for the ellipsoids enclosing those masses and plotted in the same colour, respectively. The bottom panel plots absolute values of the three direction cosines of the different major axes. The black line is computed from $\hat{a}_{1}$ of the CG and the DM halo at the same epoch $t$. The red and blue lines are computed from $\hat{a}_{1}$ defined at $t$ and the present epoch $t_{0}$ for the CG and the DM halo, respectively.

We choose five redshifts (indicated by the vertical dotted lines) to investigate the snapshots in more detail; before and after two major merger events $(z=1.49,1.31,067$, and $0.52)$ and at present $(z \approx 0)$. The signature of the mergers is clearly seen in the top panel of Figure 1, where the DM halo mass significantly increases. The first and second columns in Figure 2 show the surface density of dark matter component and the corresponding ellipsoids projected along the $z$-axis, respectively. At each redshift, we extract a cube of $(5 \mathrm{pMpc})^{3}$ around the centre of the CG of that halo. The white squares in the first column indicate the box square in the second column. Similarly, we extract a cube of $(100 \mathrm{pkpc})^{3}$ around the centre of the CG, and plot the surface density of stellar component and the corresponding ellipsoids in the third and fourth columns, respectively.

Figure 1 indicates that masses, axis ratios, and orientations of those objects did not change much after the last major merger around $8 \mathrm{Gyr}$. Before the epoch, the axis ratios and the orientations change significantly, presumably due to 


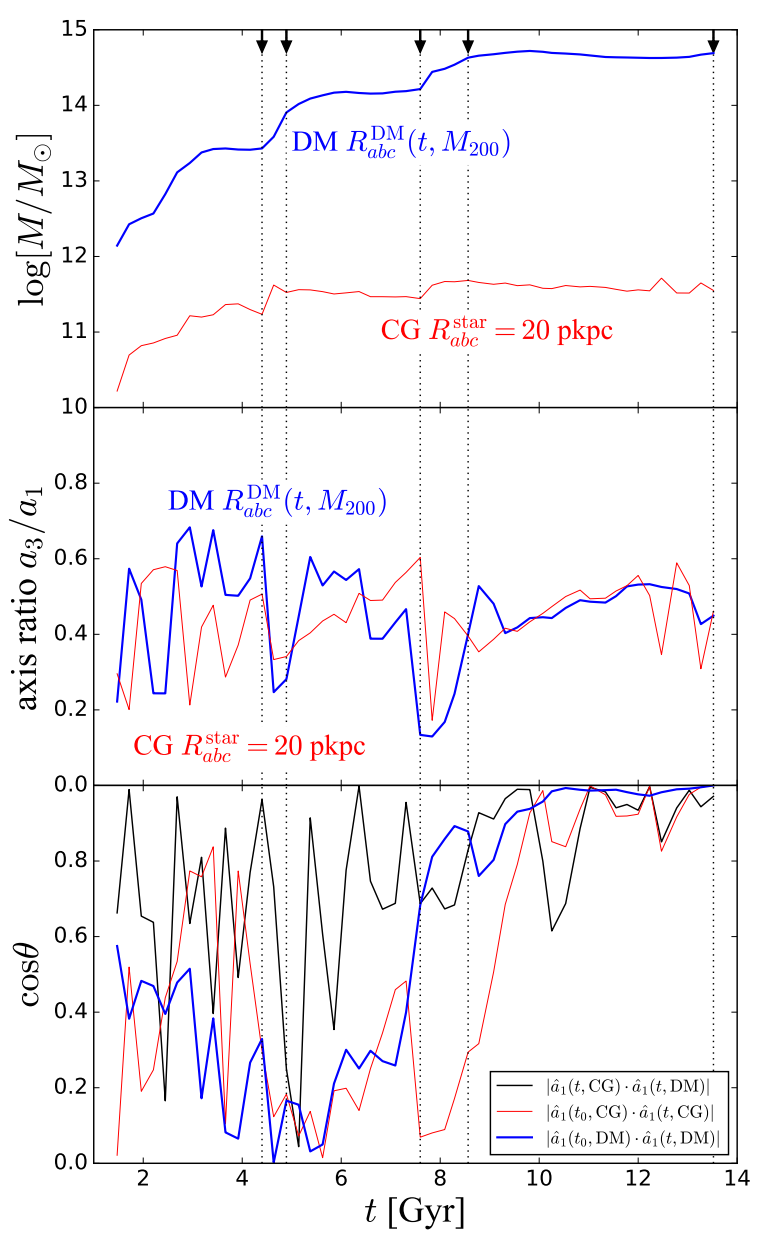

Figure 1. Top: The redshift evolution of the DM mass $M_{200}$ (thick blue) and mass of the CG within 20 pkpc (thin red) for an example of one halo shown in Figure 2. Vertical dotted lines correspond to five epochs shown in Figure 2. Middle: The redshift evolution of major-to-minor axis ratios $a_{3} / a_{1}$ of fitted ellipsoids both for the DM and for the CG. Bottom: Alignment angles between orientations of the CG and the DM halo at each epoch (black), orientations of the DM at the present epoch and in the past (thick blue), and orientations of the CG at the present epoch and in the past (thin red).

repeated mergers or mass accretion events during the growth of the halo. In particular, the shape of the DM halo became very elongated at the two major merger events, leading to rapid changes of $a_{1}$ during the mergers. This also leads to the enhancement of the angular momentum amplitude during the merger episode (Peirani et al. 2004). While there are large variations between the orientations of the $\mathrm{CG}$ and the host DM halo, they are relatively well aligned at each epoch (black line in the bottom panel), and evolve coherently toward their current direction (blue and red lines).

The above features are visually illustrated in Figure 2. The major merger between $z=1.49$ and 1.31 proceeded through the mass accretion along the upper-left to lowerright filamentary structure. Thus the major axes of the DM haloes and CG follow the direction of the filament and do not change much, even though their ellipticities, in particular at the outer boundary, significantly change during the merger event. A similar trend is seen at the next major event between $z=0.67$ and 0.52 .

After $z=0.52(t=8.5 \mathrm{Gyr})$, the DM halo did not experience any violent merger (see the top panel of Figure 1), and the axis ratio and direction of the major axis of the outer boundary of the DM halo (corresponding to $M_{200}$ ) are fairly constant until the present epoch. The orientations of the inner DM haloes defined at $0.1 M_{200}$ and $0.5 M_{200}$ and the CG gradually became aligned toward that of the outer DM halo.

The evolution history of this specific halo presented in Figures 1 and 2 is summarized as follows. The DM halo grows through sequences of repeated mergers and mass accretions following the surrounding large-scale structure, in particular along the nearby filamentary structures. The shape and orientation of the DM halo are significantly affected by those events, whereas they did not evolve much after the last major merger around $8 \mathrm{Gyr}$. The inner part of the DM halo and CG evolves rather coherently so that their major axes become aligned better toward that of the outer DM halo, which is basically fixed just after the last major event.

It is not clear, however, to what extent the above simple picture is applicable to other DM haloes and CGs as well in general. Therefore we analyse the orientations of all the 40 haloes and study the statistical evolution behavior in the next section. 


$$
z \text {-projection }
$$

$\mathrm{DM}, 5 \mathrm{pMpc}$
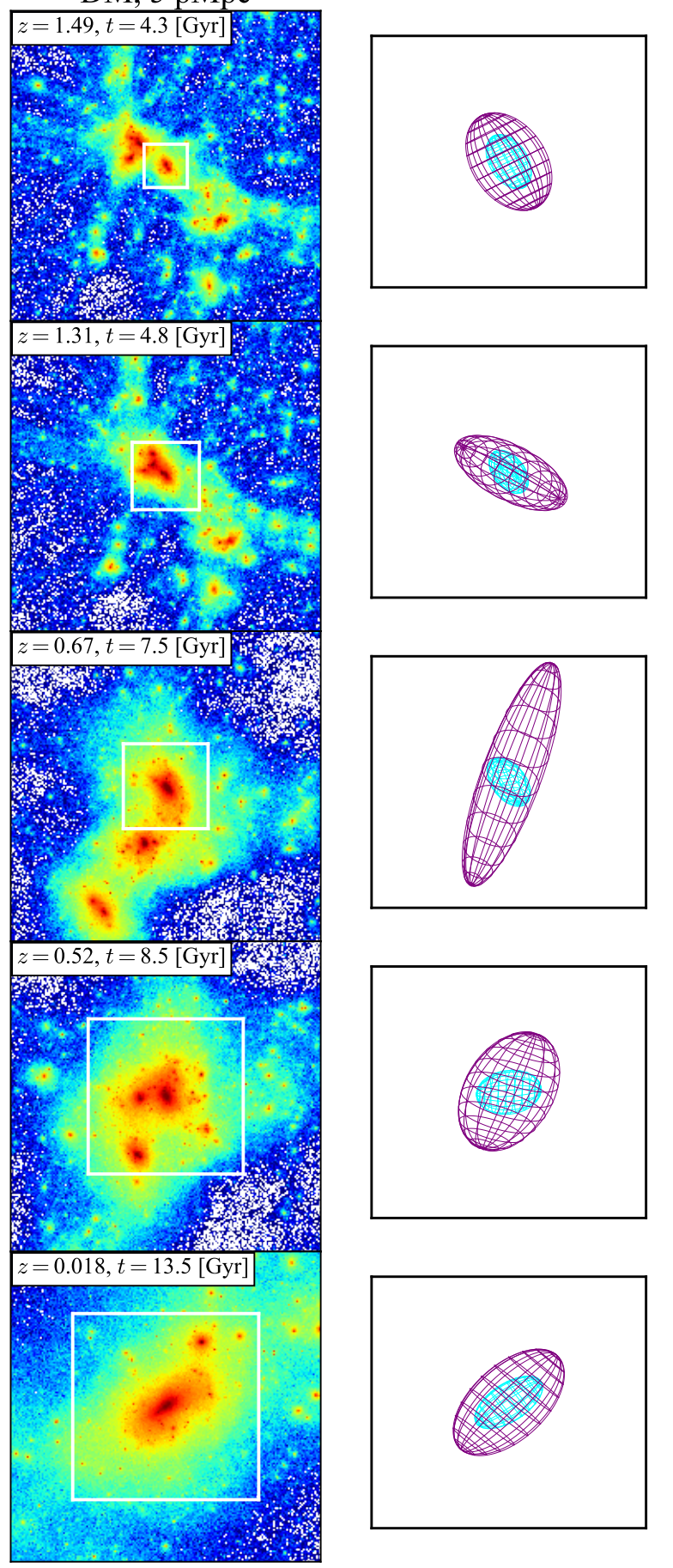

$\operatorname{DM}\left(M_{200}\right)$

$\operatorname{DM}\left(0.5 M_{200}\right)$
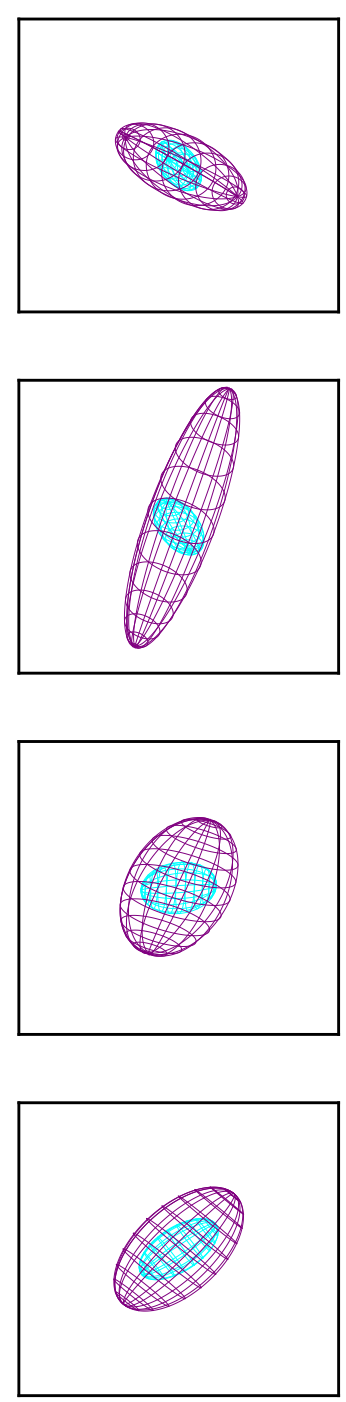

star, 100 pkpc

$\mathrm{DM}\left(0.1 M_{200}\right)$

CG $R_{a b c}^{\mathrm{star}}=20 \mathrm{pkpc}$
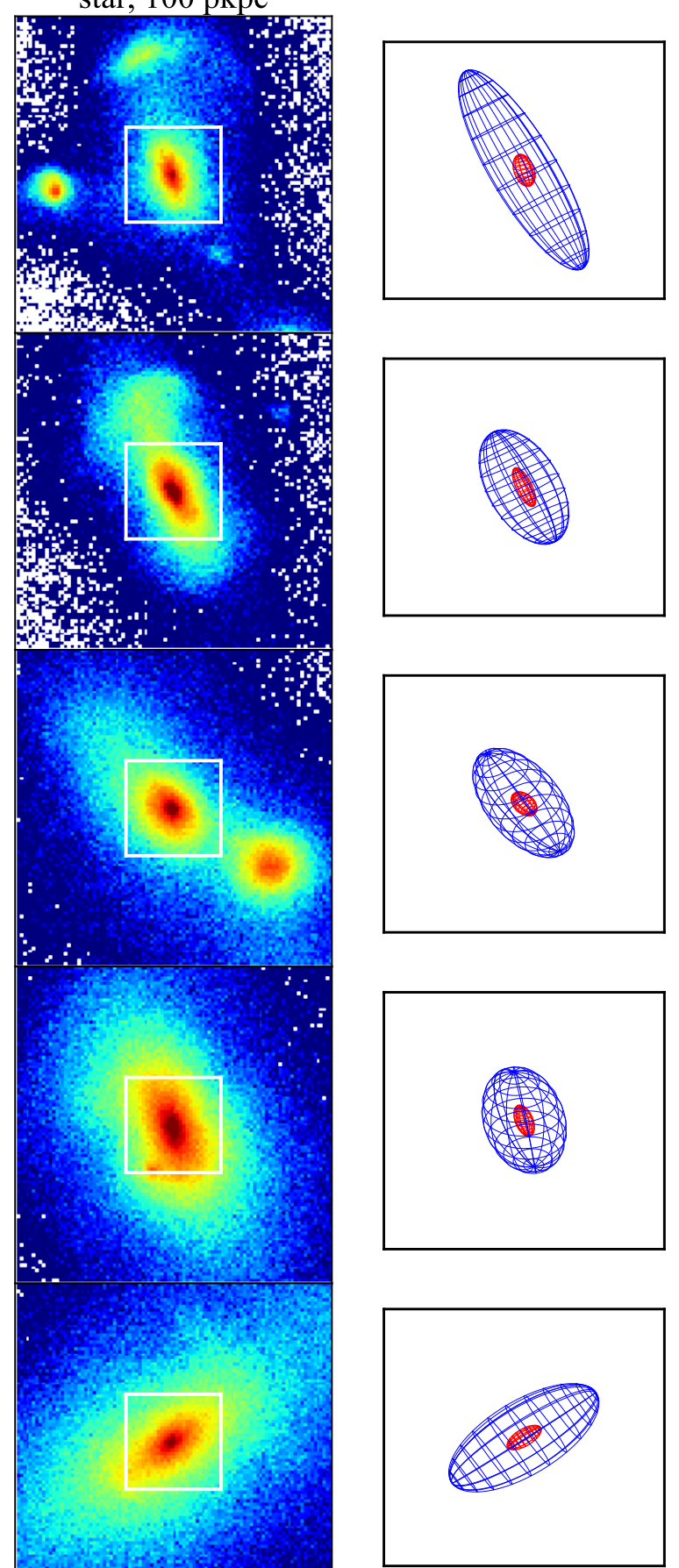

Figure 2. From left to right, we show images projected along the $z$-direction of the Horizon-AGN simulation box of DM particles within a $(5 \mathrm{pMpc})^{3}$ cube, fitted ellipsoids of DM for the enclosed mass of $M_{200}$ (purple) and $0.5 M_{200}$ (cyan), stellar particles within a (100 pkpc) ${ }^{3}$ cube, and the ellipsoids of the DM for $0.1 M_{200}$ (blue) and CG (red), respectively. From top to bottom, the images correspond to those at $z=1.49(t=4.3 \mathrm{Gyr}), z=1.31(t=4.8 \mathrm{Gyr}), z=0.67(t=7.5 \mathrm{Gyr}), z=0.52(t=8.5 \mathrm{Gyr})$, and $z=0.018(t=13.5 \mathrm{Gyr})$, respectively. These five epochs are also indicated by vertical dotted lines in Figure 1. 


\section{STATISTICAL CORRELATION AMONG ORIENTATIONS OF DM HALOES, CGS, AND SURROUNDING TIDAL FIELD}

In order to examine the validity of a simple picture emerging from the evolution of the particular halo presented in the previous section, we consider three different aspects of the statistical correlation over 40 simulated haloes; (i) instantaneous correlation of orientations between CGs and DM haloes, (ii) evolution of the orientation of CGs and DM haloes towards their present values, and (iii) statistical correlation and evolution of their orientation with respect to the surrounding tidal field. As we will show below, those results indicate that the orientations of DM haloes at the present epoch are basically imprinted in the initial conditions of the large-scale structure, while the orientations of CGs drastically evolve with time due to mergers and mass accretions.

\subsection{Instantaneous correlation of orientations between the CGs and DM haloes}

We first examine to what extent the orientations of CGs are aligned to that of the host DM haloes instantaneously. For that purpose, we compute the direction cosines between the unit vectors along the major axes of CGs and DM haloes at the same epoch, and then average them over the entire 40 haloes:

$\langle\cos \theta\rangle(t ; \mathrm{CG}-\mathrm{DM}) \equiv \frac{1}{N_{\mathrm{cl}}} \sum_{i=1}^{N_{\mathrm{cl}}}\left|\hat{a}_{1}^{(i)}(t, \mathrm{CG}) \cdot \hat{a}_{1}^{(i)}(t, \mathrm{DM})\right|$.

Figure 3 plots equation (7) for CGs against their host DM haloes defined at the mass scale of $M_{200}$ (blue-solid line) and $0.1 M_{200}$ (cyan-dotted line). Since equation (7) should reduce to 0.5 (or $\cos ^{-1}(0.5)=60^{\circ}$ ) if the two major axes are uncorrelated and randomly oriented, Figure 3 indicates that the major axes of CGs are always positively aligned to those of their host DM haloes. In order to see the evolution of the above alignment more clearly, we plot their cumulative probability density functions in Figure 4 at 50 epochs. The alignment between CGs and their host DM haloes becomes more tightly aligned toward the present epoch.

As expected, CGs are correlated more strongly with the inner part of the DM haloes at any epoch, with a mean relative angle less than $\cos ^{-1}(0.8) \approx 40^{\circ}$. This result is qualitatively consistent with the observational claim by West et al. (2017) that orientations of BCGs and their host DM haloes are aligned even at $z>1.3(t<5 \mathrm{Gyr})$. It is not easy, however, to compare our results with West et al. (2017) quantitatively, partly because cluster masses of the West et al. (2017) sample are $M_{\text {vir }} \sim 10^{15} M_{\odot}$, whereas masses of our sample are $M_{200} \sim 10^{14} M_{\odot}$. We also find that the correlation with DM haloes increases gradually on average toward the present epoch. The average alignment angles between CGs and the outer boundary of DM haloes at $M_{200}$ are $\approx \cos ^{-1}(0.70)=45^{\circ}$ before $t=8 \mathrm{Gyr}$ and $\approx \cos ^{-1}(0.82)=35^{\circ}$ at present (see also Paper II), respectively.

Since the angles are observationally measurable only in the projected two dimensional plane, Figure 5 compares the cumulative distribution of the angles defined in three dimensional space (see Figure 3) with those similarly defined after projected along either $x, y$, or $z$ direction in the simulation coordinates at $z \approx 0$. This plot helps understanding the connection between the three dimensional angles studied in this paper and observable two dimensional angles.

\subsection{Evolution of orientations of CGs and DM haloes towards the present time}

We consider next how the orientations of CGs and DM haloes become aligned towards their present values. Figure 6 plots

$\langle\cos \theta\rangle\left(t, t_{0} ; \mathrm{X}\right) \equiv \frac{1}{N_{\mathrm{cl}}} \sum_{i=1}^{N_{\mathrm{cl}}}\left|\hat{a}_{1}^{(i)}(t, \mathrm{X}) \cdot \hat{a}_{1}^{(i)}\left(t_{0}, \mathrm{X}\right)\right|$

for the three components, $\mathrm{X}=\mathrm{CG}$ (red-thin solid) and $\mathrm{DM}$ haloes of $0.1 M_{200}$ (cyan-dashed) and $M_{200}$ (blue-solid).

Orientations of the major axes of those objects at early epochs ( $t \leq 4$ Gyr) are quite different from the ones at the present time; the average alignment angles $\theta\left(t, t_{0}\right)$ are somewhere between $50^{\circ}$ and $60^{\circ}$, corresponding to $\cos ^{-1}(0.6)$ and $\cos ^{-1}(0.5)$. This result confirms the scenario presented in Section 3: orientations of both DM haloes and CGs change drastically with time. The correlation of each component increases gradually and steadily toward the present epoch, in particular, at $t>8 \mathrm{Gyr}$.

Since Figure 6 may suggest a possible break of the correlation curves around $t=8 \mathrm{Gyr}$, we examined both the occurrence rate of the last major merger events and the cluster mass growth history for the 40 haloes individually. However, they seem to be fairly continuous around $t=8 \mathrm{Gyr}$, and therefore we do not think that this epoch has any particular physical meaning. On the other hand, it corresponds approximately to the median epoch when the mass of each cluster exceeds the half of its current value. This may explain why orientations of both DM haloes and CGs remain close to their present ones at $t>8 \mathrm{Gyr}$.

Figure 6 also appears to indicate that the orientations of the outer DM haloes first become aligned closer to its present value, followed by that of the inner DM haloes, and then by that of CGs. This result suggests that the alignment proceeds from larger to smaller scales. Therefore those orientations and their mutual alignment may be determined by the surrounding larger-scale structure.

Figure 6 implies that the change of orientations between DM haloes and CGs is driven by strong dynamical interactions through successive mergers and mass accretion episodes. To check this point more explicitly, in Figure 7 we show the correlation between fractional mass changes and changes of orientations at neighboring snapshots with a time interval of $\Delta t=250 \mathrm{Myr}$. Figure 7 indicates that changes of orientations are large when fractional mass differences are large, which correspond to mergers and large mass accretions, both for DM haloes and CGs. This suggests that the spin swings of both DM haloes and galaxies are mainly driven by their mergers and mass accretions, while they are also affected by the later re-distribution of the angular momentum vector inside them. This picture is qualitatively consistent with the result of Welker et al. (2014). 


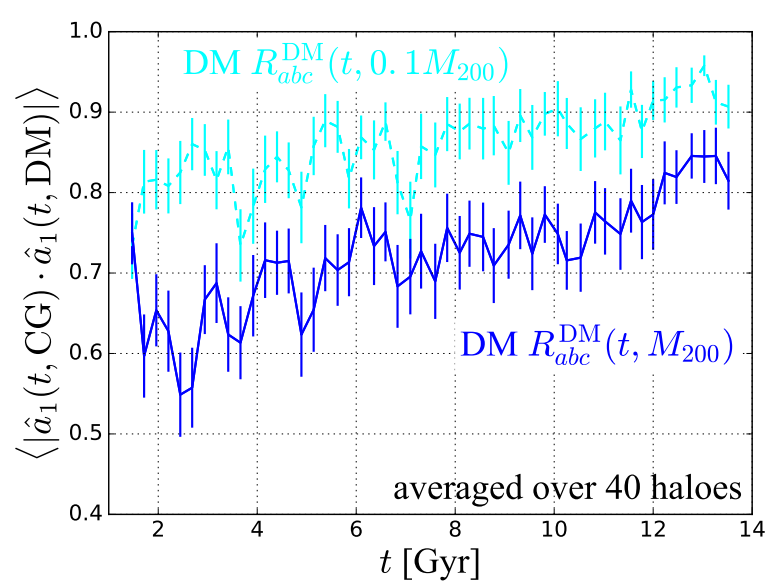

Figure 3. Correlation between orientations of CGs and DM haloes evaluated at the same epoch. Dashed cyan and solid blue lines indicate the direction cosine between CGs and DM haloes for enclosed masses of $0.1 M_{200}$ and $M_{200}$, respectively, averaged over 40 haloes. The error bars correspond to the determination accuracy of the mean values defined as the standard deviation divided by the square root of the number of haloes, $N_{\mathrm{cl}}=40$.

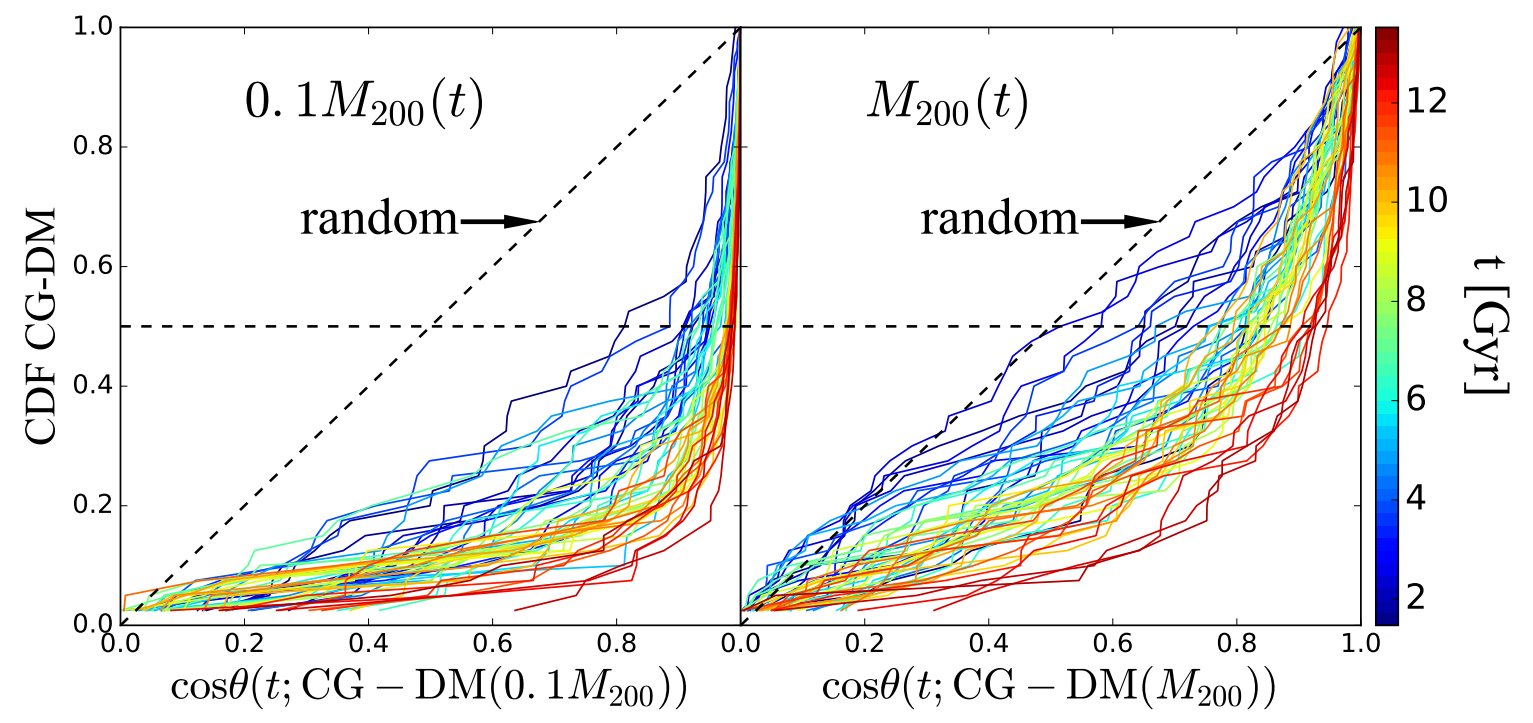

Figure 4. Cumulative probability distributions of alignment angles between orientations of DM haloes and CGs at each epoch $t$. Left and right panels show results for DM haloes with the enclosed mass $0.1 M_{200}$ and $M_{200}$, respectively. Colour scale corresponds to the cosmic time, bluer lines are earlier and redder lines are later.

\subsection{Orientations of DM haloes and CGs with respect to the surrounding large-scale structure}

The results presented in the previous subsections imply that the large scale environment is responsible for the orientations and the alignments of CGs and their host DM haloes. Thus we choose the orientations of the eigenvectors of the tidal field as a proxy of the directions embedded in the large scale structure, which may keep the memory of the initial conditions.

Figure 8 plots the correlation of the three eigenvectors $\hat{u}_{\alpha}$ computed at each epoch $(t)$ and the present epoch $\left(t_{0}\right)$. We apply three different smoothing lengths, $\sigma=3 h^{-1}, 5 h^{-1}$, and $10 h^{-1} \mathrm{cMpc}$, and compute the eigenvectors at the loca- tion of CGs according to the procedure described in Section 2.3. As is clear from Figure 8, those eigenvectors do not change so much over the cosmic time.

In particular, directions of the tidal field eigenvectors with $\sigma=10 h^{-1} \mathrm{cMpc}$ are fairly constant over $\sim 10 \mathrm{Gyr}$. Since $10 h^{-1} \mathrm{cMpc}$ is sufficiently larger than the size of the typical cluster-sized haloes and less than the typical separation $\left(\sim 30 h^{-1} \mathrm{cMpc}\right)$ of the nearest cluster-sized halo, we choose $10 h^{-1} \mathrm{cMpc}$ as the smoothing length in the following analysis, and adopt $\hat{u}_{\alpha}\left(t_{0} ; \sigma=10 h^{-1} \mathrm{cMpc}\right)$ defined at the CG's location as a set of proxies for the preferential directions imprinted in the large-scale structure surrounding those haloes.

In order to see the relation of the orientations of objects 


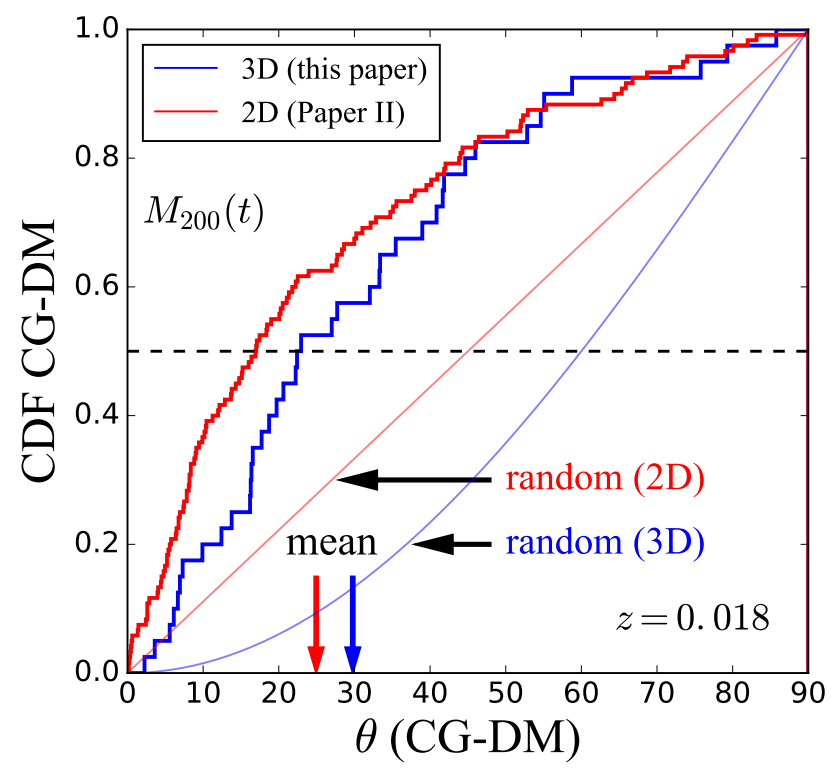

Figure 5. Cumulative probability distributions of alignment angles $\theta$ between orientations of DM haloes and CGs at present epoch $z=0.018$. Blue and red thick lines correspond to the alignment angles of three dimensional (3D) fit (this paper) and two dimensional (2D) fit (Paper II), respectively. The angles $\theta$ of 3D fit is the same as the right panel in Figure 4. The angles $\theta$ of $2 \mathrm{D}$ fit is the same of the bottom right panel in Figure 6 of Paper II. Red and blue thin lines correspond to the cumulative probability distribution of the random distributions. Mean values of $\theta$ for both the 3D and 2D are shown with arrows.

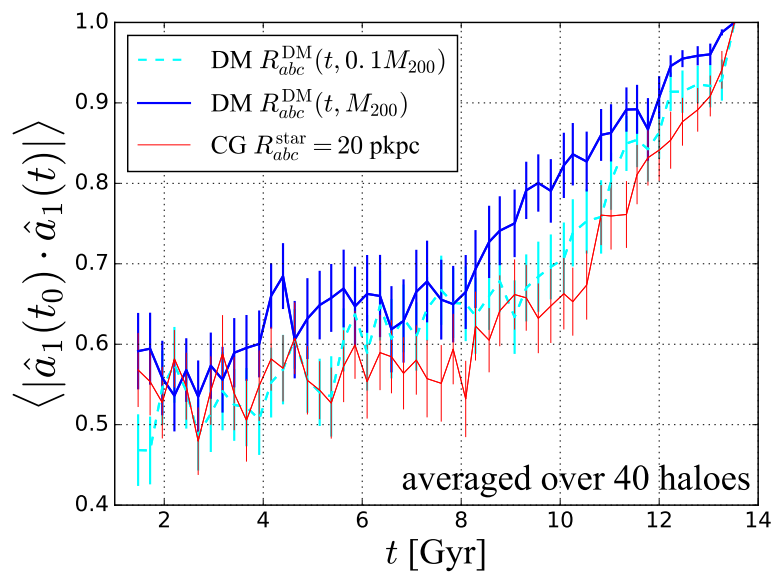

Figure 6. Correlation between orientations of objects at $t$ and the present epoch $t_{0}$ for the three components; Red-thin line is for CGs, and cyan-dashed and blue-solid lines are for DM haloes with enclosed masses of $0.1 M_{200}$ and $M_{200}$, respectively. The quoted error-bars represent the root mean square value divided by $\sqrt{N_{\mathrm{cl}}}$.

and the surrounding environment, we compute the correlations of the major axis direction of $\hat{a}_{1}(t ; \mathrm{X})$, where $X=\mathrm{CG}$, inner DM halo, and outer DM halo, against the eigenvectors of the tidal field $\hat{u}_{\alpha}\left(t_{0}\right)$ averaged over the 40 halo locations. Figure 9 plots $\left\langle\left|\hat{a}_{1}(t) \cdot \hat{u}_{\alpha}\left(t_{0}\right)\right|\right\rangle$ as a function of $t$ for $\alpha=1,2$, and 3 in the left, centre, and right panels, respectively. Each panel has three curves corresponding to the three objects; CG (red), inner DM halo (cyan) and outer DM halo (blue). The major axes of the three objects exhibit positive and negative correlations with $\hat{u}_{3}\left(t_{0}\right)(\sim 0.6)$ and $\hat{u}_{1}\left(t_{0}\right)(\sim 0.4)$, respectively, relative to the random distribution. The inter- mediate axis of the tidal field, on the contrary, is almost uncorrelated $(\sim 0.5)$ with the major axis of the objects, although they tend to become weakly negative correlated gradually toward the present epoch $(\sim 0.4)$.

In order to see the evolution of the above alignment more clearly, we plot the cumulative probability density functions in Figure 10. The upper and lower panels show those for DM haloes and CGs against $\hat{\boldsymbol{u}}_{1}$ (left), $\hat{\boldsymbol{u}}_{2}$ (centre), and $\hat{\boldsymbol{u}}_{3}$ (right). Each curve represents the cumulative probability density function at $t$ according to the colour-bar shown to the right. The diagonal dotted line indicates the 


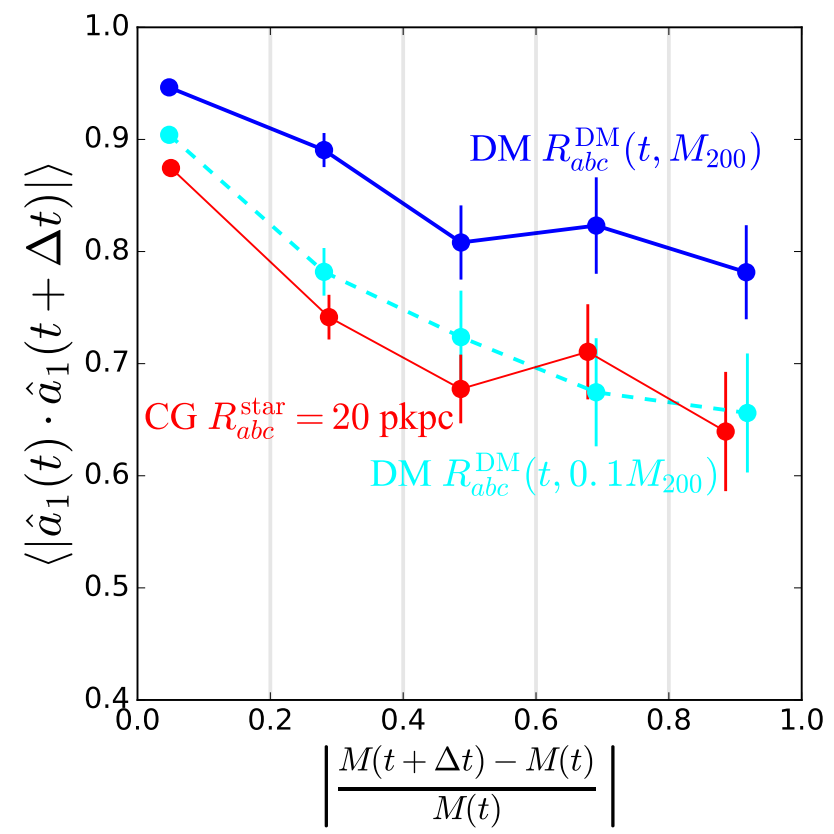

Figure 7. Correlations between changes of orientations and those of masses during the time interval $\Delta t=250 \mathrm{Myr}$. We take the absolute values of the fractional mass differences because large negative values correspond to flyby galaxies that are below the detection threshold of the galaxy finder and detach after their passage, and therefore negative values are similar to mergers with large positive values. For each bin of the fractional mass difference, we show the average and error of direction cosines of major axes of neighboring epochs. To compute the averages and the errors, we use all the 40 haloes and 49 snapshot pairs for each halo. The quoted error-bars represent the root mean square value divided by the square root of the number of corresponding objects in each bin. Red-thin line is for CGs, and cyan-dashed and blue-solid lines are for DM haloes with enclosed masses of $0.1 M_{200}$ and $M_{200}$, respectively.

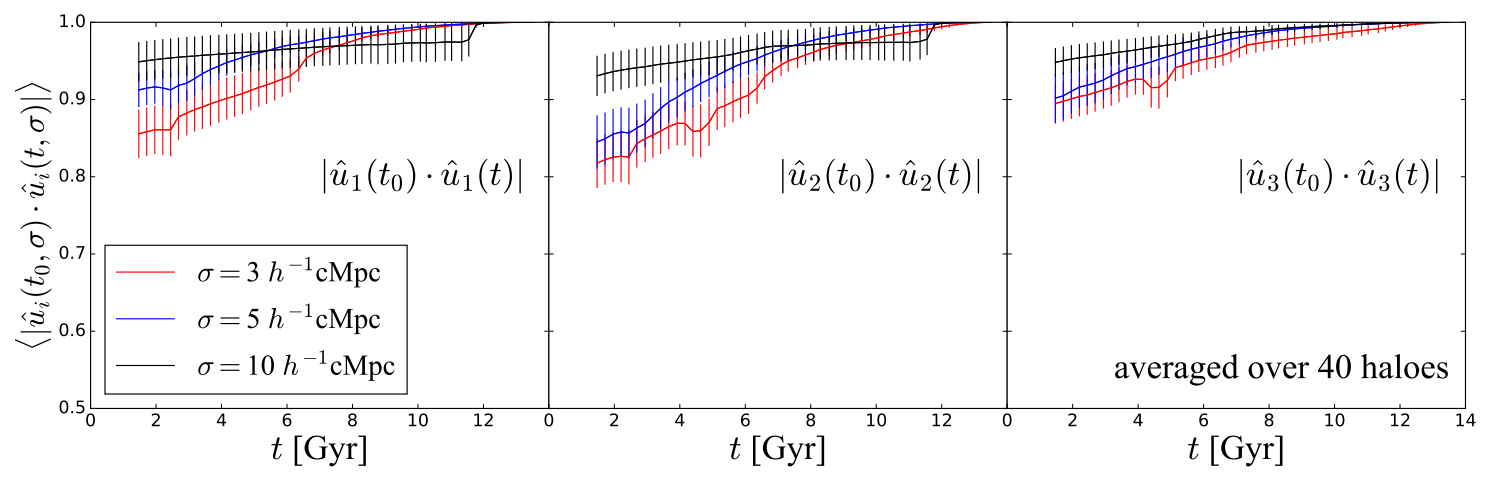

Figure 8. Correlation between the eigenvectors of the tidal field at $t$ and the present epoch $t_{0}$. They are computed from a density field Gaussian-smoothed over $\sigma=3 h^{-1}$ (red), $5 h^{-1}$ (blue), and $10 h^{-1}$ (black) cMpc; see Section 2.3 for further details. The eigenvectors are labelled as $\hat{u}_{1}, \hat{u}_{2}$, and $\hat{u}_{3}$ corresponding to the largest, medium, and smallest eigenvalues. Their correlations $\left|\hat{u}_{\alpha}\left(t_{0}\right) \cdot \hat{u}_{\alpha}(t)\right|$ averaged over the 40 halo locations are plotted for $\alpha=1,2$ and 3 in the left, centre and right panels, respectively. The quoted error-bars represent the root mean square value divided by $\sqrt{N_{\mathrm{cl}}}$. The sudden change at $\sim 12 \mathrm{Gyr}$ in the left and middle panels is due to an outlier cluster whose eigenvectors suddenly change at that epoch.

completely random distribution. Positive and negative correlations correspond to the convex and concave curves in Figure 10, respectively.

As we have seen in Figure 9, the major axes of DM haloes evolve preferentially toward the direction of $\hat{\boldsymbol{u}}_{3}\left(t_{0}\right)$. The major axes of DM haloes tend to be away from $\hat{\boldsymbol{u}}_{1}\left(t_{0}\right)$ in a time-independent manner. They are fairly uncorrelated with $\hat{\boldsymbol{u}}_{2}\left(t_{0}\right)$ at the early epochs, but develop weak correlation toward the present epoch. The correlation of CGs against the tidal field are weaker than that of DM haloes, but exhibits qualitatively a similar trend. This is consistent with the fact that 11 and 29 out of our 40 clusters correspond to "clusters" and "filaments", respectively, according to the definition in Section 2.3 (e.g. Hahn et al. 2007).

Bate et al. (2019) have studied in particular the evolution of alignments of massive elliptical galaxies relative to the tidal field. They find that the alignments are tighter for $\hat{u}_{1}$ and $\hat{u}_{3}$ than for $\hat{u}_{2}$, and also that the alignments increase from $z=3$ to 0 . These two findings are consistent with our results. 


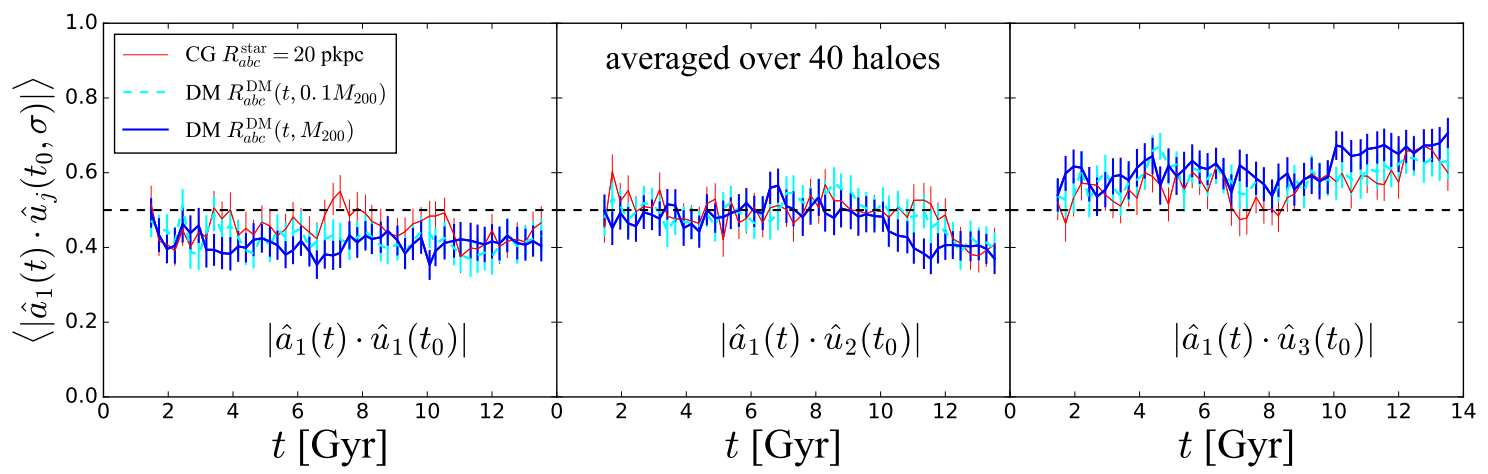

Figure 9. Mean values of alignment angles between orientations of haloes at each epoch $t$ and eigenvectors of the tidal field at the present epoch $t_{0}$. Left, middle, and right panels show the alignments of halo orientations with respect to eigenvectors $\hat{u}_{1}, \hat{u}_{2}$, and $\hat{u}_{3}$, respectively. Dashed cyan, thick blue, and thin red lines indicate median alignment angles of DM haloes for enclosed masses of $0.1 M_{200}$, $M_{200}$, and those of CGs, respectively. The quoted error-bars represent the root mean square value divided by $\sqrt{N_{\mathrm{cl}}}$. The smoothing scale of the tidal field is set to $\sigma=10 h^{-1} \mathrm{cMpc}$.

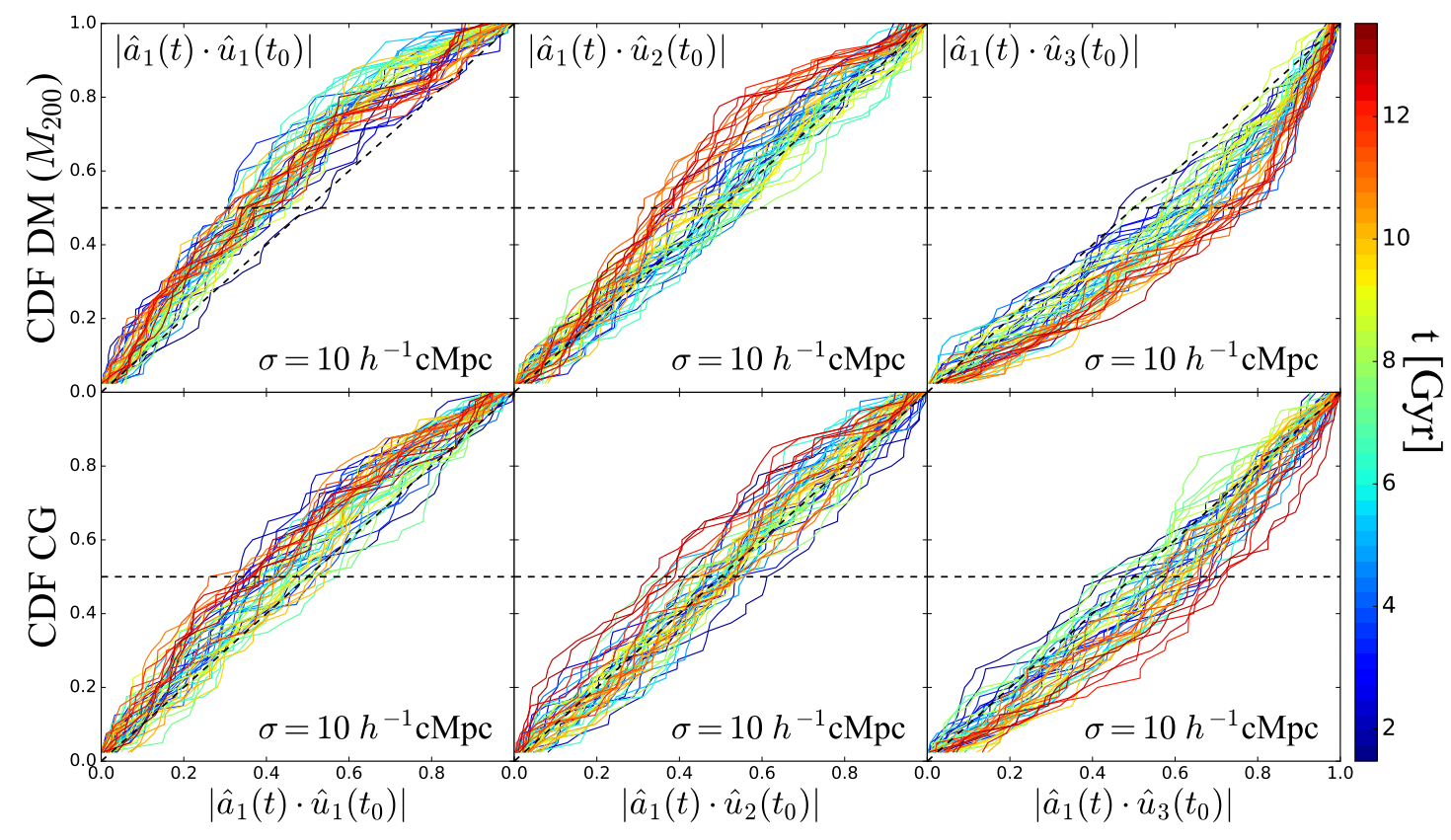

Figure 10. Cumulative probability distributions of alignment angles between orientations of haloes at each epoch $t$ and eigenvectors of the tidal field at the present epoch $t_{0}$. Top and Bottom panels show results for dark matter haloes with the enclosed mass $M_{200}$ and for CGs, respectively. Left, middle, and right panels show the position angles of $\hat{u}_{1}, \hat{u}_{2}$, and $\hat{u}_{3}$ relative to $a_{1}(t$, DM), respectively. Colour scale corresponds to the cosmic time, bluer lines are earlier and redder lines are later. The smoothing scale of the tidal field of $\sigma=10$ $h^{-1} \mathrm{cMpc}$ is adopted.

\section{SUMMARY}

This paper has examined the correlation of orientations of the central galaxies (CGs) and their host dark matter (DM) haloes extracted from the Horizon-AGN cosmological hydrodynamical simulation (Dubois et al. 2014). We identified 40 cluster-sized DM haloes at $z \approx 0$, and traced their progenitor haloes and CGs at 50 different redshifts up to $z \sim 5$. By applying the three-dimensional ellipsoidal fitting to those objects, we adopted the direction of their major axes $\hat{a}_{1}(t)$ as a measure representing their orientations. In addition, we computed the eigen-vectors of the tidal field centred at the location of the $\mathrm{CG}$ in each halo, and found that $\hat{u}_{3}(t)$ smoothed over $10 h^{-1} \mathrm{cMpc}$ corresponding to the direction of the slowest collapsing (or even stretching) mode is a good proxy characterising the orientation of the large-scale structure surrounding each object. 


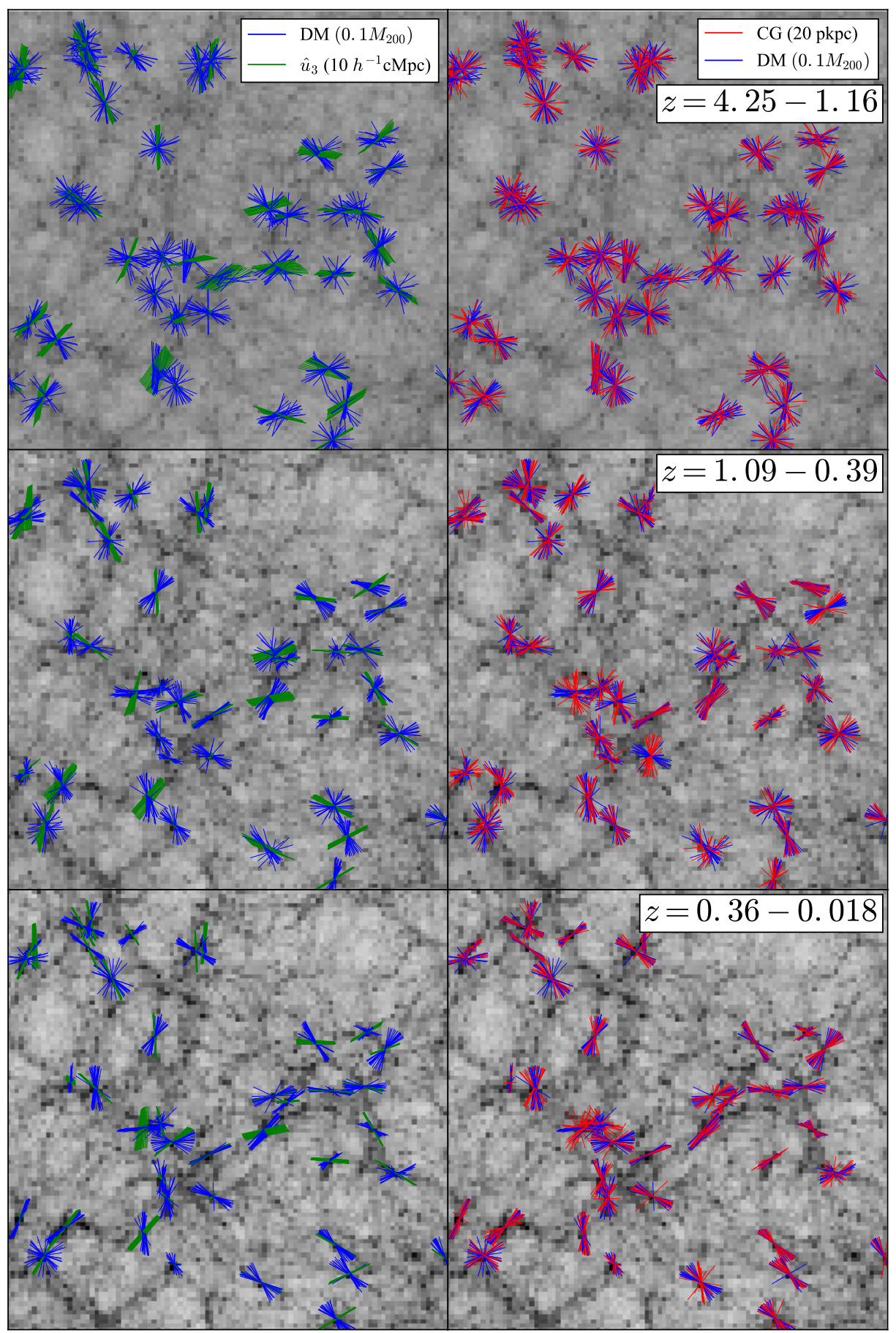

Figure 11. Projected mass density fields of DM component and the orientations of CGs (red), DM haloes for the enclosed mass of $0.1 M_{200}$ (blue), and the tidal field eigenvectors $\hat{u}_{3}$ (green) for early (top panel, $z=4.25-1.16, t=1.5-5.4$ Gyr), middle (middle panel, $z=1.09-0.39, t=5.6-9.6 \mathrm{Gyr}$ ), and late (bottom panel, $z=0.36-0.018, t=9.8-13.5 \mathrm{Gyr}$ ) epoch. In each panel, all the eigenvectors in the redshift range are shown. The size of each panel corresponds to the simulation box size, $100 \mathrm{~h}^{-1} \mathrm{cMpc}$. Lengths of lines indicate orientations with respect to the projection, long lines are nearly perpendicular to the line of sight and short lines are nearly parallel to the line of sight, respectively. Grey scales correspond to the surface mass density of DM component which are computed by the projection of all particles in the simulation box at middle time for each panel $t=1.97$ (top), $t=0.67$ (middle), $t=0.16$ (bottom) Gyr, respectively. 
A picture of the evolution of the orientations of CGs and DM haloes emerging from our current study is summarized as follows. Even at early epochs $(t<4$ Gyr $)$, orientations of the CG and its host DM halo in an individual system exhibit significant correlation in a statistical sense. The orientations of the $\mathrm{CG}$ and host DM halo are well aligned at each epoch, and their alignment becomes tighter toward the present epoch. On the other hand, the orientations of both the $\mathrm{CG}$ and its host DM halo significantly change due to mergers and continuous mass accretions; the orientations of the CG and host DM halo change coherently, and evolve together toward their current orientations that are more tightly correlated with the surrounding large-scale matter distribution $\hat{u}_{3}\left(t_{0}\right)$ than at early epochs. This implies that the instantaneous alignment between the DM halo and the $\mathrm{CG}$ is driven by strong dynamical interactions through repeated mergers and mass accretions. Since the direction of $\hat{u}_{3}(t)$ barely changes over the cosmic time, the current orientation of the DM halo, and therefore that of the CG, is basically imprinted in the primordial density field of the Universe. Indeed the CG evolves following that of the host DM halo and becomes tightly aligned with each other; their typical angles are $<30^{\circ}$ and $<20^{\circ}$ in the three dimensional space and in the projected plane, respectively, at the present epoch.

The above basic picture is visually illustrated in Figure 11. Each panel depicts the simulation box of $\left(100 h^{-1} \mathrm{cMpc}\right)^{3}$ projected along the $z$-axis of the simulation. The grey scale represents the surface density of DM component on $\left(1 h^{-1} \mathrm{cMpc}\right)^{2}$ cells at $z=1.97$ (top), 0.67 (centre) and 0.16 (bottom). Green bars in the left panels and red bars in the right panels indicate the eigen-vector $\hat{u}_{3}(t)$ of the tidal field and the major axis $\hat{a}_{1}(t)$ of CGs projected on each $x-y$ plane, whereas blue bars in all the panels are the projected major axis $\hat{a}_{1}(t)$ of DM haloes at epochs around the redshift of each panel. The green bars are roughly aligned along the filamentary structure and do not change so much. The blue bars seem to be aligned with the green bars gradually with time, and the tendency of the mutual alignment is stronger between the blue and red bars, i.e., DM haloes and CGs.

In this paper, we presented the predicted evolution of alignments between BCGs, DM haloes, and the large-scale structure, which should be confronted with observations. A caveat is that we focused on the evolution of the same halo over the cosmic time whose mass is different at different epochs (see Figure 1). Such difference of masses should be taken into account for a fair comparison with observations (Lin et al. 2017). The survey result by Hyper Suprime-Cam Survey (Aihara et al. 2018) would be useful for examining the redshift evolution of the alignment between orientations of BCGs and clusters because it covers a large $\left(\sim 1000 \mathrm{deg}^{2}\right)$ and deep $(z \sim 1.1)$ area (Oguri et al. 2018).

\section{ACKNOWLEDGEMENTS}

We thank J. Devriendt and the Horizon-AGN team for making their simulation data available to us. We thank N. E. Chisari for sharing the draft of Bate et al. (2019) with us and also for many useful comments. We also thank S. Codis for useful comments. T.O. is supported by Advanced Leading Graduate Course for Photon Science (ALPS) at the University of Tokyo. This work is supported partly by Japan Society for the Promotion of Science (JSPS) Coreto-Core Program âĂIJInternational Network of Planetary SciencesâĂİ, and also by JSPS KAKENHI Grant Numbers JP17J05056 (T.O.), JP17K14273 (T.N.), JP18K03693 (M.O.), JP15H05892 (M.O.), JP18K03704 (T.K.), and JP18H01247 and JP19H01947 (Y. S.). T.N. acknowledges Japan Science and Technology Agency (JST) CREST Grant Number JPMJCR1414.

\section{REFERENCES}

Aihara H., et al., 2018, PASJ, 70, S8

Aubert D., Pichon C., Colombi S., 2004, MNRAS, 352, 376

Bate J., Chisari E. N., Codis S., Martin G., Dubois Y., Devriendt J., Pichon C., Adriann. S., 2019, MNRAS, submitted

Binggeli B., 1982, A\&A, 107, 338

Blazek J., McQuinn M., Seljak U., 2011, J. Cosmology Astropart. Phys., 5, 010

Blazek J., Vlah Z., Seljak U., 2015, J. Cosmology Astropart. Phys., 8, 015

Blazek J., MacCrann N., Troxel M. A., Fang X., 2017, arXiv eprints,

Brunino R., Trujillo I., Pearce F. R., Thomas P. A., 2007, MNRAS, 375, 184

Carter D., Metcalfe N., 1980, MNRAS, 191, 325

Catelan P., Kamionkowski M., Blandford R. D., 2001, MNRAS, 320, L7

Chen S., Wang H., Mo H. J., Shi J., 2016, ApJ, 825, 49

Chen Y.-C., Ho S., Blazek J., He S., Mandelbaum R., Melchior P., Singh S., 2019, MNRAS, 485, 2492

Chisari N., et al., 2015, MNRAS, 454, 2736

Chisari N., et al., 2016, MNRAS, 461, 2702

Chisari N. E., et al., 2017, MNRAS, 472, 1163

Codis S., et al., 2015, MNRAS, 448, 3391

Codis S., Jindal A., Chisari N. E., Vibert D., Dubois Y., Pichon C., Devriendt J., 2018, MNRAS, 481, 4753

Dong X. C., Lin W. P., Kang X., Ocean Wang Y., Dutton A. A., Macciò A. V., 2014, ApJ, 791, L33

Dubinski J., 1998, ApJ, 502, 141

Dubois Y., et al., 2014, MNRAS, 444, 1453

Dubois Y., Peirani S., Pichon C., Devriendt J., Gavazzi R., Welker C., Volonteri M., 2016, MNRAS, 463, 3948

Durret F., Tarricq Y., Márquez I., Ashkar H., Adami C., 2019, A\&A, 622, A78

Faltenbacher A., Jing Y. P., Li C., Mao S., Mo H. J., Pasquali A., van den Bosch F. C., 2008, ApJ, 675, 146

Frigo M., 1999, in Proc. 1999 ACM SIGPLAN Conf. on Programming Language Design and Implementation. ACM, pp $169-180$

Frigo M., Johnson S. G., 1998, in Proc. 1998 IEEE Intl. Conf. Acoustics Speech and Signal Processing. IEEE, pp 1381-1384

Frigo M., Johnson S. G., 2005, Proceedings of the IEEE, 93, 216

Hahn O., Carollo C. M., Porciani C., Dekel A., 2007, MNRAS, 381,41

Hao J., Kubo J. M., Feldmann R., Annis J., Johnston D. E., Lin H., McKay T. A., 2011, ApJ, 740, 39

Hirata C. M., Seljak U., 2004, Phys. Rev. D, 70, 063526

Hirata C. M., Mandelbaum R., Ishak M., Seljak U., Nichol R., Pimbblet K. A., Ross N. P., Wake D., 2007, MNRAS, 381, 1197

Huang H.-J., Mandelbaum R., Freeman P. E., Chen Y.-C., Rozo E., Rykoff E., Baxter E. J., 2016, MNRAS, 463, 222

Jing Y. P., Suto Y., 2002, ApJ, 574, 538

Kaviraj S., et al., 2017, MNRAS, 467, 4739

Lee J., 2019, ApJ, 872, 37 
Lin Y.-T., et al., 2017, ApJ, 851, 139

Oguri M., et al., 2018, PASJ, 70, S20

Okabe T., Nishimichi T., Oguri M., Peirani S., Kitayama T., Sasaki S., Suto Y., 2018, MNRAS, 478, 1141

Okumura T., Nishimichi T., Umetsu K., Osato K., 2017, arXiv e-prints,

Osato K., Nishimichi T., Oguri M., Takada M., Okumura T., 2018, MNRAS, 477, 2141

Panko E., Juszczyk T., Flin P., 2009, AJ, 138, 1709

Patiri S. G., Cuesta A. J., Prada F., Betancort-Rijo J., Klypin A., 2006, ApJ, 652, L75

Peirani S., Mohayaee R., de Freitas Pacheco J. A., 2004, MNRAS, 348, 921

Peirani S., et al., 2017, MNRAS, 472, 2153

Peirani S., et al., 2019, MNRAS, 483, 4615

Piras D., Joachimi B., Schäfer B. M., Bonamigo M., Hilbert S., van Uitert E., 2018, MNRAS, 474, 1165

Sastry G. N., 1968, PASP, 80, 252

Smargon A., Mandelbaum R., Bahcall N., Niederste-Ostholt M., 2012, MNRAS, 423, 856

Suto D., Kitayama T., Nishimichi T., Sasaki S., Suto Y., 2016, PASJ, 68, 97

Suto D., Peirani S., Dubois Y., Kitayama T., Nishimichi T., Sasaki S., Suto Y., 2017, PASJ, 69, 14

Tenneti A., Mandelbaum R., Di Matteo T., Kiessling A., Khandai N., 2015, MNRAS, 453, 469

Tweed D., Devriendt J., Blaizot J., Colombi S., Slyz A., 2009, A\&A, 506, 647

Velliscig M., et al., 2015, MNRAS, 453, 721

Wang Y., Yang X., Mo H. J., Li C., van den Bosch F. C., Fan Z., Chen X., 2008, MNRAS, 385, 1511

Welker C., Devriendt J., Dubois Y., Pichon C., Peirani S., 2014, MNRAS, 445, L46

Welker C., et al., 2019, arXiv e-prints, p. arXiv:1909.12371

West M. J., 1994, MNRAS, 268, 79

West M. J., Villumsen J. V., Dekel A., 1991, ApJ, 369, 287

West M. J., de Propris R., Bremer M. N., Phillipps S., 2017, Nature Astronomy, 1, 0157

van Uitert E., Joachimi B., 2017, MNRAS, 468, 4502

This paper has been typeset from a $\mathrm{TE}_{\mathrm{E}}$ /LATEX file prepared by the author. 